\title{
Cavitation in Amorphous Solids
}

\author{
Pengfei Guan, ${ }^{1}$ Shuo Lu, ${ }^{2}$ Michael J. B. Spector, ${ }^{1}$ Pavan K. Valavala, ${ }^{1}$ and Michael L. Falk ${ }^{1,3,4}$ \\ ${ }^{1}$ Materials Science and Engineering, Johns Hopkins University, Baltimore, Maryland 21218, USA \\ ${ }^{2}$ Materials Science and Engineering, Beijing University of Aeronautics and Astronautics, Beijing 100191, China \\ ${ }^{3}$ Mechanical Engineering, Johns Hopkins University, Baltimore, Maryland 21218, USA \\ ${ }^{4}$ Physics and Astronomy, Johns Hopkins University, Baltimore, Maryland 21218, USA
}

(Received 11 June 2012; published 30 April 2013)

\begin{abstract}
Molecular dynamics simulations of cavitation in a $\mathrm{Zr}_{50} \mathrm{Cu}_{50}$ metallic glass exhibit a waiting time dependent cavitation rate. On short time scales nucleation rates and critical cavity sizes are commensurate with a classical theory of nucleation that accounts for both the plastic dissipation during cavitation and the cavity size dependence of the surface energy. All but one parameter, the Tolman length, can be extracted directly from independent calculations or estimated from physical principles. On longer time scales strain aging in the form of shear relaxations results in a systematic decrease of cavitation rate. The high cavitation rates that arise due to the suppression of the surface energy in small cavities provide a possible explanation for the quasibrittle fracture observed in metallic glasses.
\end{abstract}

DOI: 10.1103/PhysRevLett.110.185502

PACS numbers: 63.50.Lm, 62.20.mm, 64.70.pe

Amorphous materials, commonly termed glasses when quenched from the melt, occur in every class of material including ceramics, metals, and polymers. While the shear response of amorphous solids has received a significant amount of attention in the theoretical physics and molecular simulation literature over the past decade [1-8], significantly less attention has been devoted to hydrostatic loading in such systems $[9,10]$. This omission appears significant since experimental studies in metallic glass (MG) and other amorphous solids reveal nanocavities $[11,12]$ that form during or subsequent to deformation and strongly implicate cavitation in the physics of the fracture process zone, even when the fracture behavior is relatively brittle [13-15]. The importance of cavitation in fracture is supported by recent molecular dynamics (MD) simulations in glassy $\mathrm{Cu}_{50} \mathrm{Zr}_{50}$ and $\mathrm{Fe}_{80} \mathrm{P}_{20}$ [16].

Theory and simulation studies have been applied to understand this process in liquids more commonly than in glasses. Two recent studies [17,18] simulated the process of homogeneous nucleation in liquids in comparison with experimental data. These studies observed evidence of the curvature dependence of the surface energy on the measured cavitation rate, an effect that has itself been the subject of a significant amount of study [19-22]. One particularly notable contribution from simulation was the mapping out of the point at which the gas-liquid spinodal dips below the glass line and the glass must become unstable to cavitation [23].

Continuum mechanics approaches to modeling the kinetics of cavitation have been developed over many years

Published by the American Physical Society under the terms of the Creative Commons Attribution 3.0 License. Further distribution of this work must maintain attribution to the author(s) and the published article's title, journal citation, and DOI. by numerous researchers [6,24-30]. Often preexisting cavities are assumed to exist due to voids, inclusions, or intersections of grains, and the effect of surface energy is neglected. Various assumptions have been considered regarding the plastic constitutive behavior around the cavity $[6,24-30]$. In these cases, because plastic and elastic energy both scale with the volume of the void, above a critical stress cavity growth becomes unbounded. Here, as in some earlier work in the literature [31], we consider the effect of surface energy, and take an explicitly statistical view of cavitation so as to develop an expression for the cavitation rate that allows us to make direct connection to our simulation results.

We begin by considering the expected nucleation rate of cavities in an amorphous solid. We then compare our simulation results in $\mathrm{Zr}_{50} \mathrm{Cu}_{50}$ to the theoretical predictions and show that a classical nucleation theory (CNT) that includes the effects of plastic flow and curvature dependent surface energy is valid on short time scales, while on longer time scales strain aging effects become important.

We start from the assumption that the energy fluctuation needed to nucleate a cavity must be sufficient to generate the new surface and also to rearrange the material to accommodate the cavity. We can express this criterion as

$$
d F=d F_{e}-d F_{s}>\delta W_{p},
$$

where $d F$ is an incremental change in free energy due to an incremental change in the size of the void, which can be decomposed into contributions from the bulk elastic response $d F_{e}$ and the surface $d F_{s}$. These must exceed $\delta W_{p}$, the incremental plastic work required for the void to grow. If the void is spherical and grows monotonically we can rewrite Eq. (1) as

$$
4 \pi r^{2} K \varepsilon_{\infty} d r-8 \pi \gamma r d r>\delta W_{p}(r, \dot{r})
$$


where $K$ is the bulk modulus, $\varepsilon_{\infty}$ is the volumetric strain at the remote boundary, and $\gamma$ is the surface energy. If we assume that the plastic dissipation scales only with the cavity volume then Eq. (2) becomes

$$
r K\left(\varepsilon_{\infty}-\varepsilon_{c}\right) d r>2 \gamma d r,
$$

where we define $\varepsilon_{c}$ via the equivalency $\delta W_{p} \equiv K \varepsilon_{c} d V_{c}$, where $V_{c}$ is the volume of the cavity. The surface energy is known to be curvature dependent at small radii, and a form for this dependence was proposed by Tolman [19] in which $\gamma=\gamma_{\infty} /(1+2 \delta / r)$. Here $\gamma_{\infty}$ is the surface energy of the flat surface and $\delta$ is a parameter known as the Tolman length. We can see that the cavity becomes unstable at a radius of

$$
r_{c}=r^{*}\left(\frac{1}{2}+\frac{1}{2} \sqrt{1+\frac{4 \delta}{r^{*}}}-\frac{2 \delta}{r^{*}}\right),
$$

where $r^{*}=2 \gamma_{\infty} / K\left(\varepsilon-\varepsilon_{c}\right)$ represents the critical radius for the case where surface energy is independent of curvature $(\delta=0)$. The free energy barrier for cavitation is

$$
\Delta F_{c}=\frac{4}{3} \pi r_{c}^{3} K\left(\varepsilon-\varepsilon_{c}\right)-4 \pi \gamma r_{c}^{2} .
$$

Assuming that this energy barrier is large compared to the thermal energy scale $k_{B} T$ the rate of cavitation is $R_{\varepsilon}=$ $\nu \exp \left(-\Delta F_{c}(\varepsilon) / k_{B} T\right)$, where $\nu$ is the attempt frequency.

To investigate the process of cavitation in MG, we carry out a series of MD simulations in simple models of two binary MGs using the LAMMPS [32] code. We employ the embedded atom method potential recently developed to describe the interactions for $\mathrm{Zr}-\mathrm{Cu}$ [33]. Two independent $\mathrm{Zr}_{50} \mathrm{Cu}_{50} \mathrm{MG}$ systems were quenched with cooling rates of $1 \times 10^{10}$ (denoted as G1) and $1 \times 10^{12} \mathrm{~K} \mathrm{~s}^{-1}$ (denoted as $G 2$ ). The instantaneous configurations at various temperatures $(60,100,180$, and $240 \mathrm{~K})$ provided the initial conditions for cavitation simulations with related temperatures. Further details of the simulation methodology can be found in the Supplemental Material including the means that were used to create the initial conditions for the mechanical tests and to measure the physical parameters for our theoretical analysis [34].

To quantify the dependence of the cavitation rate on temperature and applied strain we simulated the two samples, $G 1$ and $G 2$, at a variety of different state points. At each state point we simulated 100 independent cavitation events by starting with randomly distributed velocities drawn from a Boltzmann distribution appropriate for the temperature. We maintained each glass sample at constant hydrostatic tensile strain under adiabatic conditions until a cavity nucleation event was detected. The fraction of cavitated samples $f_{\varepsilon}^{T}(t)$ was calculated as a function of time for each strain $\varepsilon$ at each temperature $T$. Because all the simulations for each $\varepsilon$ were carried out from the same initial structure with the same thermomechanical history, the cavitation was found to preferentially occur at the same location for all simulations. If the cavitation process is a Poisson process the fraction cavitated can be expected to vary with time as $f_{\varepsilon}^{T}(t)=1-e^{-R_{\varepsilon}^{T} t}$, with a single time constant $R_{\varepsilon}^{T}$, the rate of cavitation.

As shown in Fig. 1, a plot of $\ln \left(1-f_{\varepsilon}^{T}(t)\right)$ as a function of $t$ does not behave in a linear fashion for any strain over the entire time window. This strongly suggests that the cavitation process is not a Poisson process, and other rate processes occur on time scales similar to the cavitation time. However, there does exist a time window for each applied strain over which $\ln \left(1-f_{\varepsilon}^{T}(t)\right)$ is linear in $t$ and therefore Poissonian. We can quantify this window by $\tau_{\varepsilon}$, the time at which our best short-time linear fit intersects a linear fit to the long-time data.

As shown in Fig. 2(a), the typical relationship between $\ln \left(1-f_{\varepsilon}^{T}(t)\right)$ and $t$ can be separated into three time scales: short-time $\left(t<\tau_{\varepsilon}\right)$, medium-time, and long-time $\left(t \gg \tau_{\varepsilon}\right)$. Here, we representatively select three samples that each cavitate on a different time scale. The potential energies (PE) as a function of the holding time for these three samples are shown in Fig. 2(b); the sudden decrease of PE corresponds to the cavity nucleation event. Here the initial structure is compared to the atomic configuration just prior to cavity nucleation via the local shear strain as shown in Figs. 2(c)-2(f) calculated using the technique introduced in Ref. [1]. The short-time cavitated sample [Fig. 2(c)] shows almost no strain before cavitation. The other two samples [see Figs. 2(d) and 2(e)] as well as the noncavitated sample [Fig. 2(f)] exhibit significant structural relaxation corresponding to regions of high local strain.

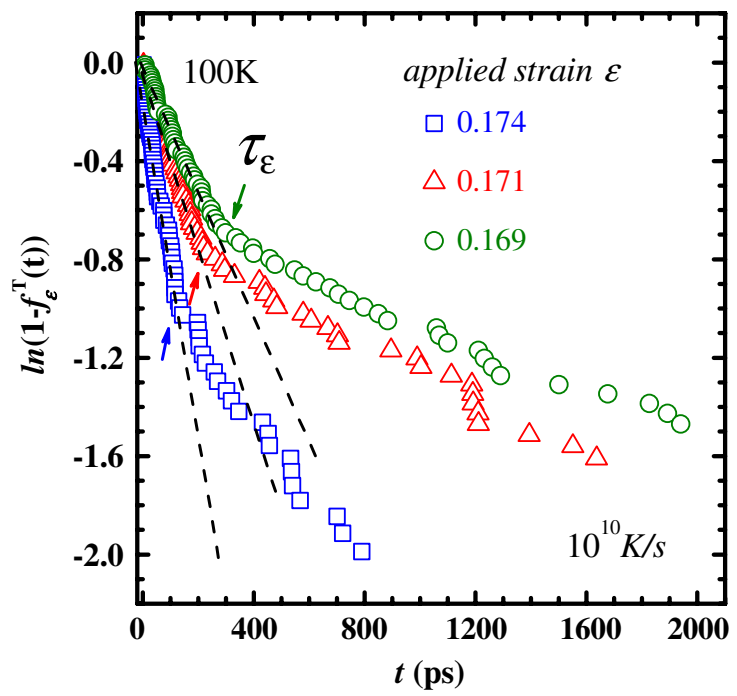

FIG. 1 (color online). The value of $\ln \left(1-f_{\varepsilon}^{T}(t)\right)$ as a function of $t$ for several applied strains of $G 1$, where $f_{\varepsilon}^{T}$ is the fraction of samples that have cavitated. The times of deviation from Poissonian behavior, $\tau_{\varepsilon}$, are denoted by arrows. The simulated temperature prior to loading is $100 \mathrm{~K}$. 

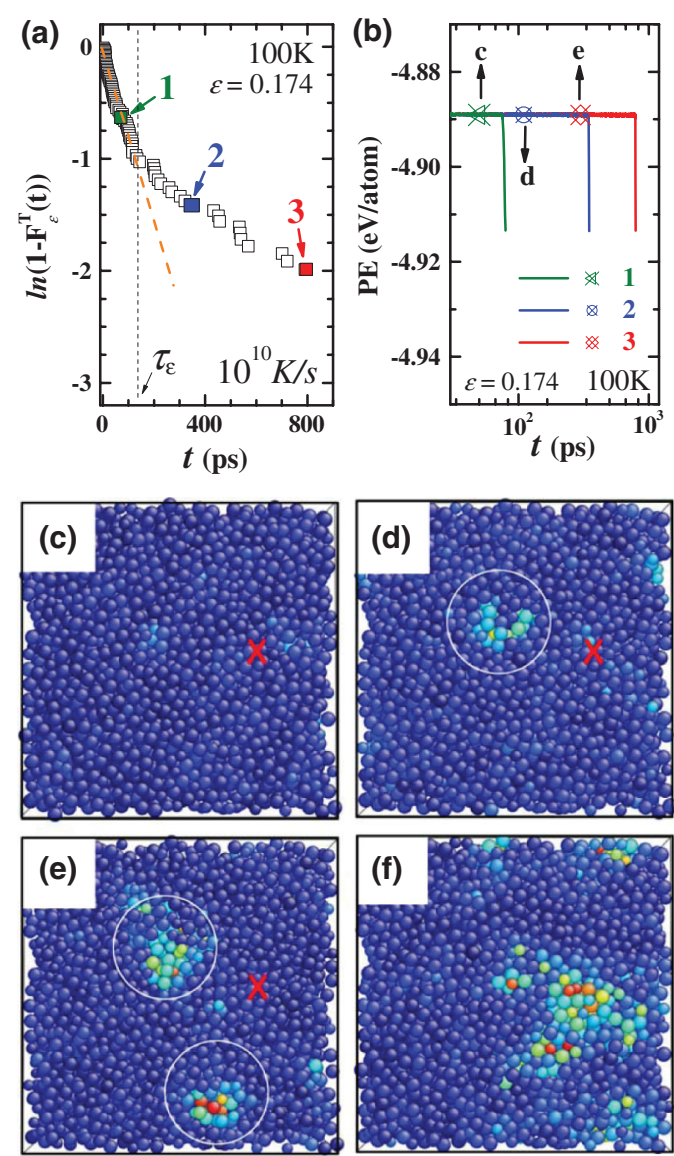

FIG. 2 (color). (a) The relationship between $\ln \left(1-f_{\varepsilon}^{T}(t)\right)$ and $t$ of $G 1$ glass at applied strain $\sim 0.174$. Three samples, marked as 1,2 , and 3 , show different cavitation times as denoted by the arrows. The potential energies as a function of holding time for these samples are shown in (b). Local strain calculations are shown at different holding times for each sample as denoted by $\mathrm{c}, \mathrm{d}$ and $\mathrm{e}$ in (b) and the local shear strains are shown in (c)-(e) for each case. The local shear strains for a noncavitated sample at $10 \mathrm{~ns}$ is shown in (f). In these images blue (black) denotes low strain $(\sim 0 \%)$ and other colors (gray) denote high strain. The white circles delineate the high strain regions and the red " $X$ " denotes the center of cavitation. The simulated temperature prior to loading is $100 \mathrm{~K}$.

The short time scale cavitation processes can be analyzed as a Poisson process and the free energy barrier to cavitation $\Delta F_{c}$ can be extracted for data from both $G 1$ and $G 2$ as shown in Fig. 3. Data from four different temperatures collapse to a single curve for each glass preparation with an attempt frequency $\nu=0.1 \pm 0.01 \mathrm{ps}^{-1}$, which is, to a good approximation, the frequency of the longest wavelength dilatational mode in the simulation cell. For fitting these data by the predictions of Eq. (5) $K$ from pressure-volume measurements was measured to be $106.0 \pm 0.2 \mathrm{GPa}$, and $\gamma_{\infty}$ from energy differences before and after the introduction of free surface was measured to be $0.080 \pm 0.02 \mathrm{eV} / \mathrm{A}^{2}$ and $0.086 \pm 0.03 \mathrm{eV} / \mathrm{A}^{2}$ for $G 1$ and $G 2$, respectively. The value of $\varepsilon_{c}, 2.2 \%$ and $2.6 \%$ for $G 1$

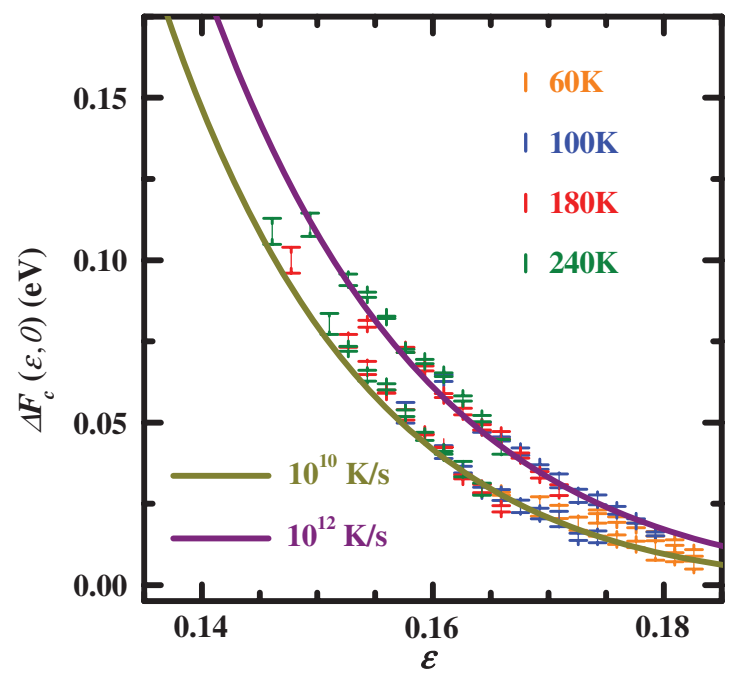

FIG. 3 (color). The free energy barrier to cavitation as a function of strain for both $G 1$ and $G 2$ at various initial simulated temperatures. Scaling is achieved with an attempt frequency of $0.1 \mathrm{ps}^{-1}$. Solid lines are plotted based on Eq. (5) with calculated parameters for each glass [34] and a Tolman length of $\delta=1.15 \AA$.

and $G 2$, respectively, was obtained from the ratio of the kinetic energy rise to the pressure drop during adiabatic cavity growth. These calculations are described in detail in the Supplemental Material [34], leaving only a single fitting parameter, the Tolman length $\delta$. Figure 3 shows the best fit for the data for both glasses with $\delta=1.15 \pm 0.01 \AA$ and implies that the short-time cavitation process can be described as the nucleation of a void with a critical size predicted by Eq. (1) and shown by the red lines in Fig. 4(a).

Because the location of cavity nucleation of each sample is now known, we can directly test the critical cavity radius prediction of the theory by embedding a bubble centered at this position and measuring the critical cavity size directly [34]. While holding the volume of the simulation cell fixed we increase the bubble radius $R$ from 0 with a constant inflation rate and measure the bubble size that induces cavitation [34]. The data are plotted against the curve extracted indirectly from the cavitation rates in Fig. 4(a). We see that the curve based on Eq. (4) with $\delta=1.15 \AA$ gives the best fitting for the directly measured $R_{c}$ data of both glasses, confirming that Eqs. (1) and (2) correctly describe the cavitation event in the $\mathrm{Cu}_{50} \mathrm{Zr}_{50}$ MG as a nucleation event. We conclude that cavitation in MG is controlled by the spatial heterogeneity in the glass with preferential nucleation sites able to cavitate by surmounting a free energy barrier that is significantly lower than would be expected in the absence of the effect of cavity radius on surface energy. The relatively smaller activation energy of the more slowly cooled MG implies that annealing can increase brittleness by lowering the barrier to cavity nucleation.

Within the CNT framework we can investigate the nature of the local fluctuations that control cavity 

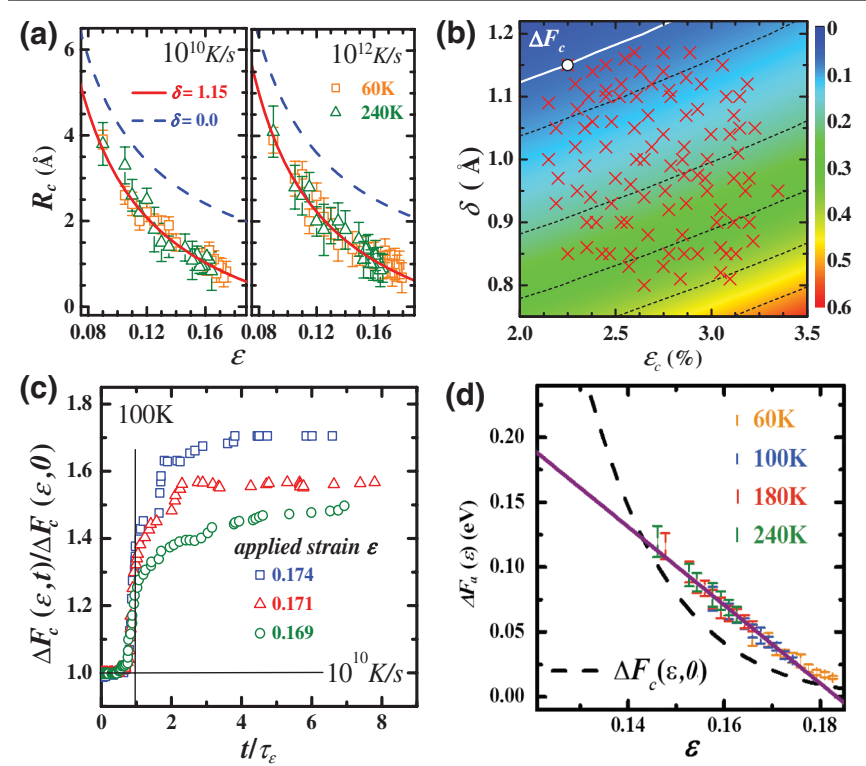

FIG. 4 (color). (a) The measured critical cavity nucleation size as a function of $\varepsilon$. The red solid lines (blue dashed lines) are the predictions by Eq. (4) with $\delta=1.15 \AA$ ( $\delta=0 \AA)$. (b) Twodimensional plot of free energy barrier $\Delta F_{c}$ as a function of $\varepsilon_{c}$ and $\delta$ based on Eqs. (3)-(5). The measured $\varepsilon_{c}$ and $\delta$ for 100 different cavitation sites are shown as red " $X$ ". The white (solid) line is related to the spontaneous cavitation event with lowest $\Delta F_{c}$. (c) The free energy barrier to cavitation normalized by the short-time Poissonian barrier to cavitation as a function of time in units of $\tau_{\varepsilon}$ showing the increase in the cavitation barrier upon activation of shear relaxation. (d) The activation energy for shear relaxation as a function of strain in $G 1$. Scaling over several temperatures is achieved with an attempt frequency of $1.0 \mathrm{ps}^{-1}$. Dashed lines are plotted based on Eq. (5) with calculated parameters for $G 134$ and the Tolman length $\delta=1.15 \AA$. All the temperatures denote the simulated temperature prior to loading.

nucleation. While many of the parameters in the theory are global $\left(K, T, \gamma_{\infty}, \varepsilon_{\infty}\right)$ two are inherently local, the Tolman length $\delta$ that characterizes local length-scale dependent variations in surface energy, and $\varepsilon_{c}$, which characterizes local susceptibility to plastic rearrangement. To determine the relative importance of local variations of these two quantities we initiate 100 cavitation events at random locations using the embedded bubble method. For each cavitation event we directly measure $\varepsilon_{c}$ and the critical radius and infer $\delta$ and $\Delta F_{c}$. These data are shown in Fig. 4(b). As expected, the spontaneous cavitation site has a lower activation barrier than any of the induced cavitation sites. The correlation coefficient between $\varepsilon_{c}$ and $\delta$ is low $(<0.13)$, and the relative sensitivity of cavitation to variations in surface energy versus plastic response, i.e., $\left(\partial \Delta F_{c} / \partial \delta\right) \delta_{\mathrm{STD}} /\left(\partial \Delta F_{c} / \partial \varepsilon_{c}\right) \varepsilon_{c_{\mathrm{STD}}}$, is approximately 3.4. While both effects play a role in determining the nucleation barrier, local surface energy fluctuations dominate.

We now consider the effect of strain aging on cavitation. Recall that the time prior to the breakdown of Poissonian behavior, $\tau_{\varepsilon}$ is dependent on the applied strain. The free energy barrier to cavitation, as inferred from the rate of cavitation, increases by $50 \%-70 \%$ from the value predicted by Eq. (5) at $\tau_{\varepsilon}$, as shown in Fig. 4(c) for loading at $100 \mathrm{~K}$. We expect that this strain aging effect, ubiquitous in glasses, is caused by structural rearrangement prior to cavitation that arises when the holding time is comparable to the characteristic shear relaxation time of the strained MG. To test this supposition we use the measured values of $\tau_{\varepsilon}$ to extract the activation energy of the shear relaxation as a function of strain in Fig. 4(d) assuming $\tau_{\varepsilon}^{-1}=\nu_{a} \exp \left(-\Delta F_{a} / k_{B} T\right)$. The attempt frequency $\nu_{a}$, found by scaling the results from all temperatures, is on the order of the Einstein frequency as expected for a local relaxation. The activation energy for shear relaxation is nearly linear with strain extrapolating to $0.55 \mathrm{eV}$ at zero pressure and zero applied shear stress and exhibiting an activation volume with respect to pressure of $4.5 \AA^{3}$. Comparison of this extrapolation with the predicted activation energies for cavitation shown as a dashed line in Fig. 4(d) indicates that only at very high strains do we expect the energy barrier for cavitation to drop below the barrier for shear relaxation.

The direct MD simulations of fracture in Ref. [16] did not observe cavitation upon loading a crack in amorphous $\mathrm{CuZr}$, but cavitation was reported under biaxial loading. The absence of cavitation and fracture initiation in that work may be attributable to the small sample sizes attainable in MD, as the observed crack opening displacement approached the sample size.

Our primary conclusion is that in the MG systems studied cavitation appears to occur via a process that is well described by CNT, and that the spatially fluctuating curvature dependent surface energy, i.e., the Tolman length effect, significantly enhances cavitation in these materials. These nucleation events appear to be heterogeneous, as nucleation always occurs on the same site in a given sample. Previous analyses have noted that variations in the elastic modulus correlate with cavity nucleation sites in amorphous polymers [35]. Our analysis indicates that these cavitation sites are characterized by low local surface energy (high $\delta$ ) and, to a lesser degree, high structural susceptibility to plastic deformation (low $\varepsilon_{c}$ ). Strain aging also appears to be important and arises from shear relaxations. The energy barriers of these two processes were extracted from our simulations of $\mathrm{Zr}_{50} \mathrm{Cu}_{50}$. The apparent ubiquity of heterogeneous nucleation sites for cavitation provides a strong indication that nanoscale cavitation must be seriously considered as one factor, and perhaps the primary factor, contributing to the fact that many MGs exhibit quasibrittle behavior during fracture. The physics of cavitation on these smallest scales, incorporating multiaxial stress states that go beyond the current analysis, needs to be incorporated into theories of constitutive response in amorphous solids in order to provide first-principles predictions of important mechanical properties such as fracture toughness and ductility. 
We thank Mark Robbins and Konrad Samwer for helpful discussions and the Homewood High Performance Cluster for computing support. This work was supported by the National Science Foundation under Grants No. DMR0808704, No. DMR1107838, and No. OCI0963185.

[1] M. L. Falk and J. S. Langer, Phys. Rev. E 57, 7192 (1998).

[2] M. L. Falk, J. S. Langer, and L. Pechenik, Phys. Rev. E 70, 011507 (2004).

[3] A. Tanguy, F. Leonforte, and J. L. Barrat, Eur. Phys. J. E 20, 355 (2006).

[4] F. Varnik, L. Bocquet, and J. L. Barrat, J. Chem. Phys. 120, 2788 (2004).

[5] E. Bouchbinder and J. S. Langer, Phys. Rev. E 80, 031133 (2009).

[6] E. Bouchbinder, J.S. Langer, T. S. Lo, and I. Procaccia, Phys. Rev. E 76, 026115 (2007).

[7] E. Bouchbinder, J. S. Langer, and I. Procaccia, Phys. Rev. E 75, 036107 (2007); 75, 036108 (2007).

[8] A. Lemaitre and C. Caroli, Phys. Rev. Lett. 103, 065501 (2009).

[9] J. Rottler and M. O. Robbins, Phys. Rev. Lett. 95, 225504 (2005).

[10] C. Schuh and A. Lund, Nat. Mater. 2, 449 (2003).

[11] W. H. Jiang, F. E. Pinkerton, and M. Atzmon, Acta Mater. 53, 3469 (2005).

[12] J. Li, F. Spaepen, and T. C. Hufnagel, Philos. Mag. A 82, 2623 (2002).

[13] X. K. Xi, D. Q. Zhao, M. X. Pan, W. H. Wang, Y. Wu, and J. J. Lewandowski, Phys. Rev. Lett. 94, 125510 (2005).

[14] E. Bouchaud, D. Boivin, J. L. Pouchou, D. Bonamy, B. Poon, and G. Ravichandran, Europhys. Lett. 83, 66006 (2008).

[15] Z.F. Zhang, J. Eckert, and L. Schultz, Acta Mater. 51, 1167 (2003)
[16] P. Murali, T.F. Guo, Y.W. Zhang, R. Narasimhan, Y. Li, and H. J. Gao, Phys. Rev. Lett. 107, 215501 (2011).

[17] A. Y. Kuksin, G. E. Norman, V. V. Pisarev, V. V. Stegailov, and A. V. Yanilkin, Phys. Rev. B 82, 174101 (2010).

[18] Q. An, G. Garrett, K. Samwer, Y. Liu, S. V. Zybin, S. N. Luo, M. D. Demetriou, W. L. William, and W. A. Goddard, J. Phys. Chem. Lett. 2, 1320 (2011).

[19] R. Tolman, J. Chem. Phys. 17, 333 (1949).

[20] J. W. P. Schmelzer, I. Gutzow, and J. Schmelzer, J. Colloid Interface Sci. 178, 657 (1996).

[21] M. P. Moody and J. Attard, J. Chem. Phys. 115, 8967 (2001).

[22] B. J. Block, S. K. Das, M. Oettel, P. Virnau, and K. Binder, J. Chem. Phys. 133, 154702 (2010).

[23] S. Sastry, Phys. Rev. Lett. 85, 590 (2000).

[24] R. Hill, The Mathematical Theory of Plasticity (Clarendon Press, Oxford, 1950).

[25] Y. Huang, J. W. Hutchinson, and V. Tvergaard, J. Mech. Phys. Solids 39, 223 (1991).

[26] M. Ortiz and A. Molinari, J. Appl. Mech. 59, 48 (1992).

[27] K. Y. Volokh, Int. J. Solids Struct. 44, 5043 (2007).

[28] X. Y. Wu, K. T. Ramesh, and T. W. Wright, J. Mech. Phys. Solids 51, 1 (2003).

[29] E. Bouchbinder, T. S. Lo, and I. Procaccia, Phys. Rev. E 77, 025101(R) (2008).

[30] E. Bouchbinder, T. S. Lo, I. Procaccia, and E. Shtilerman, Phys. Rev. E 78, 026124 (2008).

[31] D. W. Nicholson, Acta Mech. 34, 263 (1979).

[32] S. Plimpton, J. Comput. Phys. 117, 1 (1995); http:// lammps.sandia.gov.

[33] Y. Q. Cheng, E. Ma, and H. W. Sheng, Phys. Rev. Lett. 102, 245501 (2009).

[34] See Supplemental Material at http://link.aps.org/ supplemental/10.1103/PhysRevLett.110.185502 for the details of simulations and data analysis of cavitation process for $\mathrm{Zr}_{50} \mathrm{Cu}_{50}$ and $\mathrm{Fe}_{80} \mathrm{P}_{20}$ glasses.

[35] G. N. Toepperwein and J. J. de Pablo, Macromolecules 44, 5498 (2011) 